\title{
Syntheses and Pharmacokinetic Studies of Prodrug Esters for the Development of Oral Carbapenem, L-084
}

\author{
Takeshi Isoda, Hideki Ushirogochi, Koichi Satoh, Tsuyoshi Takasaki, Itsuki Yamamura, \\ Chisato Sato, Ado Mihira, Takao Abe, Satoshi Tamai, Shigeki Yamamoto, \\ Toshio Kumagai, Yoshimitsu Nagao
}

Received: February 16, 2006 / Accepted: April 13, 2006

(C) Japan Antibiotics Research Association

\begin{abstract}
We discovered an orally active carbapenem, L084, through pharmacokinetic studies on various prodrug esters of $(1 R, 5 S, 6 S)-6-[(R)-1$-hydroxyethyl]-1-methyl-2-[1(1,3-thiazolin-2-yl)azetidin-3-yl]thio-1-carbapen-2-em-3carboxylic acid (LJC11,036). L-084 showed a strong antimicrobial activity against Gram-positive and Gramnegative bacteria and exhibited the highest intestinal absorption among synthesized prodrugs of LJC11,036.
\end{abstract}

Keywords $1 \beta$-methylcarbapenem, prodrug ester, azetidine, oral administration, pharmacokinetics

\section{Introduction}

Carbapenems are well recognized to have broad antibacterial activities, and most of the carbapenem compounds have been developed for parenteral use such as imipenem [1], panipenem [2], meropenem [3], biapenem $[4,5]$, ertapenem [6] and doripenem [7]. The development of oral carbapenems is now expected in the clinical realm because oral administration is advantageous for patients. Recently several oral carbapenems have been developed as prodrug esters, GV-118819 [8], CS-834 [9] and DZ-2640 [10] and these compounds were under clinical trials.

For the development of a new oral carbapenem, we carried out a prodrug approach [11] to optimize the oral absorbability of LJC11,036 (4), which showed a strong antimicrobial activity against Gram-positive and Gramnegative bacteria. The potently active 4 also exhibited high stability to $\beta$-lactamase and human renal dehydropeptidaseI (DHP-I). After pharmacokinetic studies in rats and chemical stability tests, L-084 (5) was selected for further development among synthesized prodrug esters of 4 .

In this paper we describe the syntheses and pharmacokinetic studies of prodrug esters of $\mathbf{4}$ for the development of a new oral carbapenem.

\section{Chemistry}

The potent LJC11,036 (4) was synthesized by condensation of enolphosphate 2 [12] and thiol compound $\mathbf{1}$ and then hydrogenolysis (Scheme 1). This antimicrobial agent 4 has an azetidine moiety at the $\mathrm{C}-2$ position of the carbapenem skeleton. Although a chiral pyrrolidine moiety was widely used as the C-2 side chain for the discovery of new carbapenems, the azetidine moiety has not been applied because of the difficulty in preparing it. So, we decided to comfort the challenge of using an achiral azetidine moiety instead of a chiral pyrrolidine moiety as the C-2 side chain on carbapenem skeleton. As shown in Scheme 2, thiol compound 1 was synthesized through a key intermediate, 3-hydroxyazetidine hydrochloride (7), that was prepared according to the literature in $48 \%$ yield from benzhydrylamine [13]. Compound $\mathbf{8}$ was obtained by the
T. Isoda (Corresponding author), H. Ushirogochi, K. Satoh, T. Takasaki, I. Yamamura, C. Sato, A. Mihira, T. Abe, S. Tamai, S. Yamamoto, T. Kumagai: Medical Research Laboratories, Wyeth K.K., 1-6-34 Kashiwa-cho, Shiki-shi, Saitama 353-8511, Japan, E-mail: isoda@konicaminolta.jp
Y. Nagao: Faculty of Pharmaceutical Sciences, The University of Tokushima, 1-78-1 Sho-machi, Tokushima-shi, Tokushima 7708505, Japan 
reaction of 7 and 2-methylthio-2-thiazoline in $92 \%$ yield. Converting the hydroxy group of $\mathbf{8}$ to a thiol group was conducted by a conventional method [14].

Various prodrug esters of $\mathbf{4}$ were prepared in order to evaluate the bioavailability in rats. The esterification of 4 was carried out by using several kinds of substituted-alkyl chloride ( $\mathrm{R}-\mathrm{Cl})$ in the presence of a quaternary ammonium salt as shown in Scheme 3 [15]. Among ten synthesized derivatives, four compounds, 5, 13, 15 and 18, were obtained as crystals that were greatly advantageous for further development because of being easy to handle and to formulate. Cyclohexyloxycarbonyloxyethyl ester 12 was obtained as a diastereo-mixture and was an amorphous powder. As compound $\mathbf{1 8}$ did not show high absorbability in rats (Table 2), it was eliminated from this development. Three of the prodrugs, $\mathbf{5}, \mathbf{1 3}$ and $\mathbf{1 5}$, were subjected to accelerated conditions to evaluate their chemical stability. Table 1 shows that each amorphous powder was less stable than corresponding crystalline powder, and the crystalline

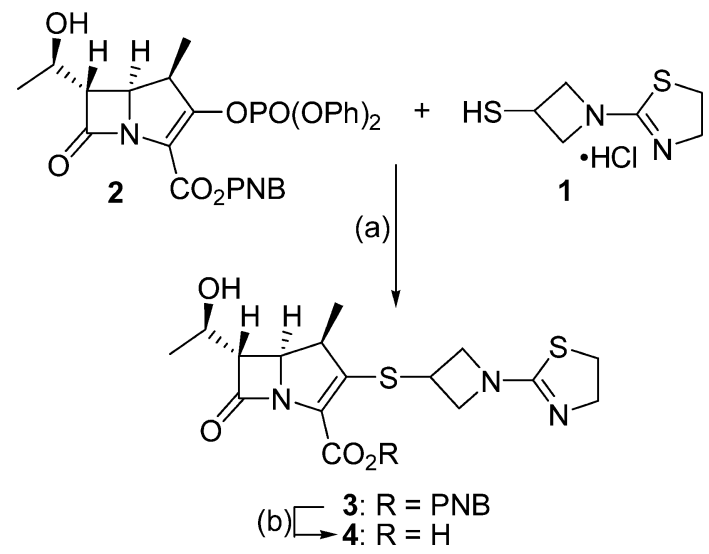

Reagents and conditions: (a) 1 (1.1 equiv.), $i-\operatorname{Pr}_{2} \mathrm{NEt}$ (2.2 equiv.), $\mathrm{MeCN},-20^{\circ} \mathrm{C}, 2$ hours, 94\%; (b) $10 \% \mathrm{Pd}-\mathrm{C}, \mathrm{NaHCO}_{3}$ (0.5 equiv.), $\mathrm{H}_{2} \mathrm{O}-n-\mathrm{BuOH}, \mathrm{H}_{2}(400 \mathrm{kPa}), 1.5$ hours, $82 \%$.

Scheme 1 forms of $\mathbf{5}$ and $\mathbf{1 3}$ were more stable than that of $\mathbf{1 5}$.

\section{Biological Properties}

As previously reported, LJC11,036 (4) exhibited strong and well-balanced activity against both Gram-positive and Gram-negative bacteria [16]. In addition to this, compound 4 showed higher stability to human renal dehydropeptidase-

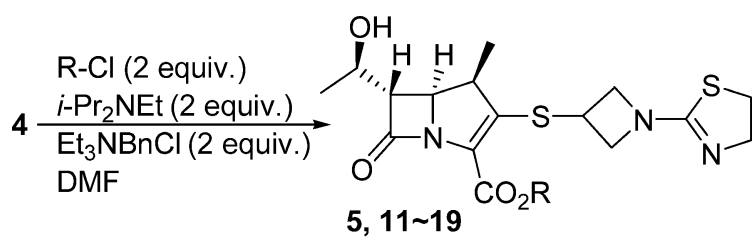

5 (L-084): $\mathrm{R}=-\mathrm{CH}_{2} \mathrm{OCOt}-\mathrm{Bu}(\mathrm{POM})$
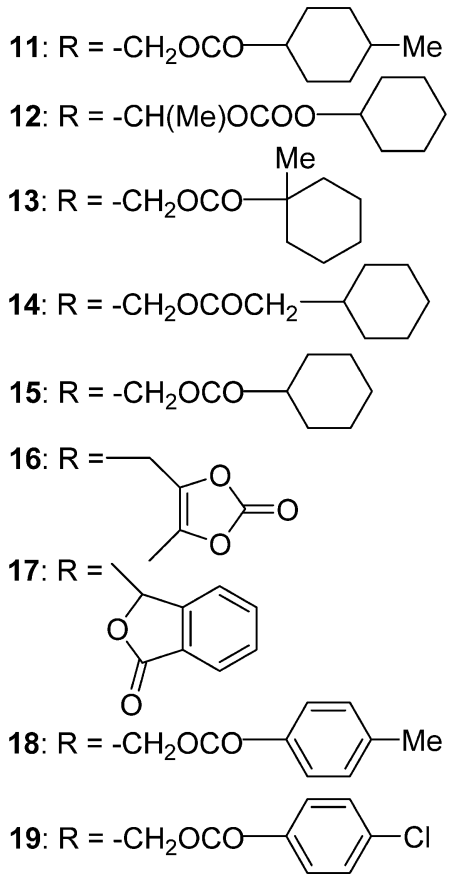

Scheme 3

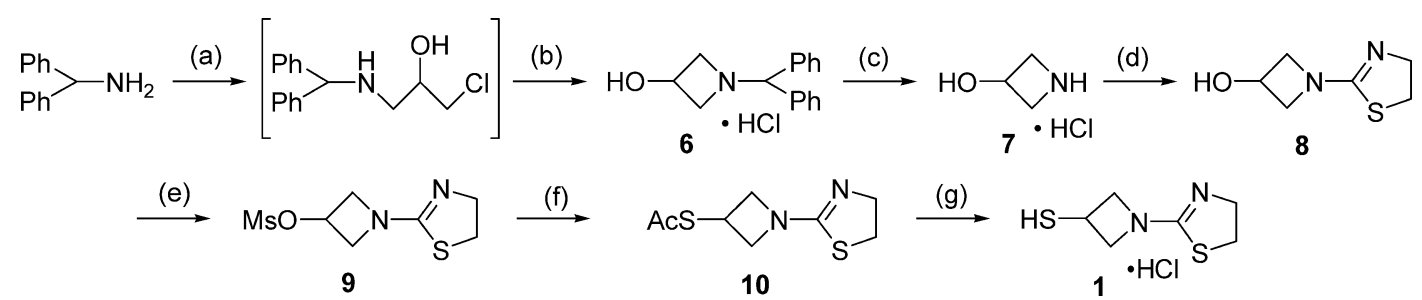

Reagents and conditions: (a) epichlorohydrin (1.0 equiv.), $\mathrm{MeOH}, \mathrm{rt}, 1$ day; (b) DMSO, 50 ${ }^{\circ} \mathrm{C}, 3$ days, $48 \%$ (2 steps); (c) $5 \%$ Pd-C, $\mathrm{H}_{2} \mathrm{O}-$ $\mathrm{EtOH}, \mathrm{H}_{2}(350 \mathrm{kPa}), \mathrm{rt}, 4$ hours, $100 \%$; (d) 2-methylthio-2-thiazoline (1.0 equiv.), $\mathrm{KHCO}_{3}(0.7$ equiv.), $\mathrm{MeOH}, \mathrm{reflux}, 1 \mathrm{day}, 92 \%$; (e) $\mathrm{MsCl}$ (1.1 equiv.), $\mathrm{Et}_{3} \mathrm{~N}$ (1.2 equiv.), DMAP (0.01 equiv.), $\mathrm{THF}, 5^{\circ} \mathrm{C}, 0.5$ hours, $93 \%$; (f) $\mathrm{AcSK}\left(1.5\right.$ equiv.), DMF, $100^{\circ} \mathrm{C}, 5.5 \mathrm{hours}, 88 \%$; (g) $\mathrm{KOH}$ (1.1 equiv.), IPA, $5^{\circ} \mathrm{C}, 10$ minutes then $\mathrm{HCl}(2.4$ equiv.), $87 \%$.

Scheme 2 
Table 1 Remaining percentage of prodrug esters after storage under accelerated conditions

\begin{tabular}{|c|c|c|c|c|c|c|}
\hline \multirow{2}{*}{$\begin{array}{l}\text { Storage conditions } \\
\text { Time (month) }\end{array}$} & \multicolumn{4}{|c|}{$40^{\circ} \mathrm{C}$} & \multicolumn{2}{|c|}{$40^{\circ} \mathrm{C}, 75 \% \mathrm{RH}^{\mathrm{a}}$} \\
\hline & 0.5 & 1 & 2 & 3 & 0.5 & 1 \\
\hline 5 amorphous & 90 & 80 & - & - & 0 & - \\
\hline crystal & 100 & 98 & - & - & 99 & 98 \\
\hline 13 amorphous & - & 68 & - & 37 & 5 & - \\
\hline crystal & - & 99 & - & 99 & - & 99 \\
\hline 15 amorphous & 85 & 71 & 48 & 38 & 0 & - \\
\hline crystal & 100 & 99 & 97 & 94 & 97 & 74 \\
\hline
\end{tabular}

${ }^{\text {a }}$ Relative humidity.

Table 2 Pharmacokinetic parameters after oral administration to rats at dose of $20 \mathrm{mg} / \mathrm{kg}$ as 4

\begin{tabular}{lcccccrrrrrrr}
\hline Compounds & $\mathbf{4}$ & $\mathbf{5}$ & $\mathbf{1 1}$ & $\mathbf{1 2}$ & $\mathbf{1 3}$ & $\mathbf{1 4}$ & $\mathbf{1 5}$ & $\mathbf{1 6}$ & $\mathbf{1 7}$ & $\mathbf{1 8}$ & $\mathbf{1 9}$ \\
\hline $\mathrm{Cmax}(\mu \mathrm{g} / \mathrm{ml})$ & 0.3 & 14.8 & 9.5 & 13.6 & 15.3 & 12.3 & 10.3 & 2.0 & 7.3 & 8.4 & 7.9 \\
$\mathrm{~A} \cup \mathrm{C}(\mu \mathrm{g} \cdot \mathrm{h} / \mathrm{ml})$ & 0.2 & 10.7 & 6.9 & 10.1 & 11.5 & 9.7 & 8.3 & 1.6 & 5.5 & 7.1 & 6.2 \\
$\mathrm{BA}(\%)^{\mathrm{a}}$ & 0.8 & 38.1 & 24.6 & 36.0 & 41.2 & 34.9 & 29.6 & 5.8 & 19.5 & 25.5 & 22.3 \\
\hline
\end{tabular}

${ }^{a}$ Bioavailability (BA) was calculated from AUC $(28 \mu \mathrm{g} \cdot \mathrm{h} / \mathrm{ml})$ after i.v. administration of $\mathbf{4}$ at a dose of $20 \mathrm{mg} / \mathrm{kg}$.

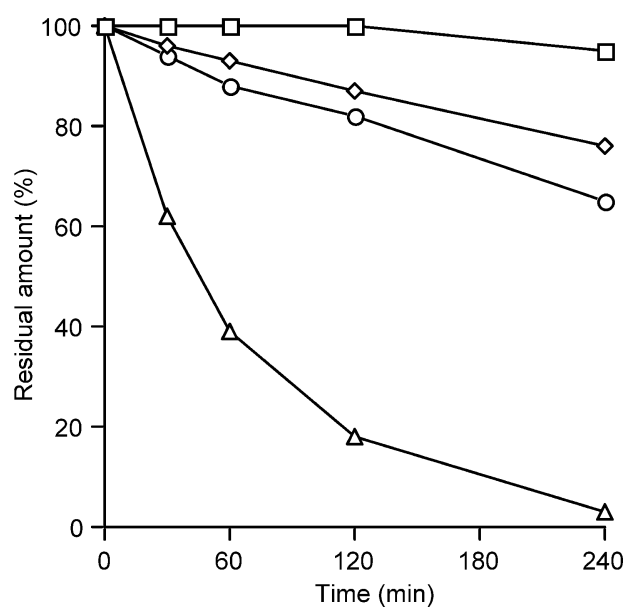

Fig. 1 Stability of LJC11,036 (4) to recombinant human DHP-I.

$\square$, Biapenem; $\diamond, L J C 11,036 ; \bigcirc$, meropenem; $\triangle$, imipenem.

I (DHP-I) than meropenem as shown in Figure 1 [17]. For the development of an orally active carbapenem, we focused on a prodrug ester of $\mathbf{4}$, which exhibited a high maximum plasma level (Cmax) of $\mathbf{4}$ in rats.
Table 3 Pharmacokinetic parameters to various species

\begin{tabular}{lcccc}
\hline \multicolumn{1}{c}{ Species } & Mouse & Dog & Monkey & Human \\
\hline Dose $(\mathrm{mg} / \mathrm{kg})^{\mathrm{a}}$ & 10 & 10 & 10 & $100 \mathrm{mg} / \mathrm{man}$ \\
Cmax $(\mu \mathrm{g} / \mathrm{ml})$ & 38.1 & 5.7 & 5.8 & 4.1 \\
AUC $(\mu \mathrm{gh} / \mathrm{ml})$ & 87.8 & 11.1 & 5.9 & 3.5 \\
Urinary recovery $(\%)$ & - & - & 25 & 72 \\
BA $(\%)$ & 71 & 35 & 45 & - \\
\hline
\end{tabular}

${ }^{a} \mathrm{~L}-084$ was orally administered to each species at a dose as an active form.

In order to evaluate oral absorption based on Cmax, AUC and BA in rats, esters of 4 were prepared (Scheme 3). The results of pharmacokinetics in rats are shown in Table 2. The Cmax of prodrugs $\mathbf{5}, \mathbf{1 2} \sim \mathbf{1 5}$ was more than $10 \mu \mathrm{g} / \mathrm{ml}$ at an oral dose of $20 \mathrm{mg} / \mathrm{kg}$ as 4 . The areas under the blood concentration-time curve (AUC) of 5, 12 and $\mathbf{1 3}$ were more than $10 \mu \mathrm{g} \cdot \mathrm{h} / \mathrm{ml}$. The BA of $\mathbf{5 , 1 2}$ and $\mathbf{1 3}$ was more than $35 \%$.

Based on these biological and chemical studies and cost of production, we chose $\mathbf{5}$ for further development. Table 3 
shows pharmacokinetic parameters of $\mathbf{4}$ after dosing $\mathbf{5}$ to mice, dogs, monkeys and humans. Especially in humans, the prodrug ester $\mathbf{5}$ showed a high Cmax and high urine excretion level of the active metabolite 4 [18]. The cumulative urinary recoveries of 4 were in the range of 65 to $79 \%$ (average: $72 \%$ ) when $\mathbf{5}$ was administered orally to healthy male volunteers at a dose corresponding to $100 \mathrm{mg}$ of 4 .

\section{Conclusion}

We found a new oral carbapenem antibiotic L-084 (5), showing a high bioavailability and Cmax in humans, by a prodrug approach. The active metabolite 4 shows potent and well-balanced antibacterial activity and also shows higher stability to DHP-I than meropenem.

\section{Experimental}

\section{General Methods}

Melting points were determined using Yanagimoto micromelting point apparatus. IR spectra were recorded on a JASCO FT-IR (VALOR-III) spectrometer. NMR spectra were performed on JEOL JNM-FX100 (100 MHz), JNMEX270 (270 MHz) or Bruker Avance DPX400 (400 MHz) spectrometers using tetramethylsilane (TMS) or sodium 3(trimethylsilyl)propionate- $d_{4}$ (TSP) as an internal standard. Mass spectra were recorded on JEOL JMS-DX300 or JMSSX102A spectrometers. Elemental analysis was recorded on Yanako CHN-Corder. HPLC analysis was performed on Shimadzu LC system constructed from SPD-10AV and LC$10 \mathrm{AS}$.

\section{1-(1,3-Thiazolin-2-yl)azetidin-3-ol (8)}

To a solution of 3-hydroxyazetidine hydrochloride (7, $39.5 \mathrm{~g}, 361 \mathrm{mmol})$ in $\mathrm{MeOH}(361 \mathrm{ml})$ were added potassium hydrogen carbonate $(25.3 \mathrm{~g}, 253 \mathrm{mmol})$ and 2-methylthio-2-thiazoline $(48.1 \mathrm{~g}, 361 \mathrm{mmol})$, and the mixture was refluxed for 23 hours. The reaction mixture was allowed to cool to $40^{\circ} \mathrm{C}$, and additional potassium hydrogen carbonate $(18.1 \mathrm{~g}, 180 \mathrm{mmol})$ was added. The mixture was stirred for 2 hours at $40^{\circ} \mathrm{C}$. After removal of insoluble material by filtration, the filtrate was concentrated under reduced pressure to give $\mathbf{8}$ as colorless crystals (52.4 g, 92\%).

Mp $114 \sim 117^{\circ} \mathrm{C}$; IR (KBr) $\mathrm{cm}^{-1} 2939,2743,1610$, 1452, 1371, 1128, 1014; ${ }^{1} \mathrm{H}-\mathrm{NMR}\left(270 \mathrm{MHz}, \mathrm{CDCl}_{3}\right)$ $\delta 3.34(2 \mathrm{H}, \mathrm{t}, J=7.4 \mathrm{~Hz}), 3.85 \sim 3.90(2 \mathrm{H}, \mathrm{m}), 3.98(2 \mathrm{H}$, $\mathrm{t}, J=7.4 \mathrm{~Hz}), 4.17 \sim 4.23(2 \mathrm{H}, \mathrm{m}), 4.62 \sim 4.71(1 \mathrm{H}, \mathrm{m})$; HRMS (EI) calcd for $\mathrm{C}_{6} \mathrm{H}_{10} \mathrm{~N}_{2} \mathrm{OS} 158.0514$, found $\mathrm{m} / \mathrm{z}$ $158.0510(\mathrm{M})^{+}$; Anal. Calcd for $\mathrm{C}_{6} \mathrm{H}_{10} \mathrm{~N}_{2} \mathrm{OS}$ : C, 45.55;
H, 6.37; N, 17.71. Found: C, 45.37; H, 6.38; N, 17.61.

3-Methanesulfonyloxy-1-(1,3-thiazolin-2-yl)azetidine (9) 1-(1,3-Thiazolin-2-yl)azetidin-3-ol $(\mathbf{8}, 27.7 \mathrm{~g}, 176 \mathrm{mmol})$ in THF $(300 \mathrm{ml})$ was stirred for 0.5 hours with DMAP $(214 \mathrm{mg}, 1.76 \mathrm{mmol}), \mathrm{Et}_{3} \mathrm{~N}(21.3 \mathrm{~g}, 211 \mathrm{mmol})$ and methanesulfonyl chloride $(22.2 \mathrm{~g}, 193 \mathrm{mmol})$ at $5^{\circ} \mathrm{C}$, and the solvent was evaporated under reduced pressure. After the addition of AcOEt $(250 \mathrm{ml})$ and a saturated aqueous solution of sodium hydrogen carbonate $(400 \mathrm{ml})$ to the residue, the mixture was stirred for 10 minutes at room temperature. The organic layer was dried over $\mathrm{MgSO}_{4}$, and the filtrate was concentrated under reduced pressure. The residue was triturated with THF $(40 \mathrm{ml})$ and $n$-hexane $(400 \mathrm{ml})$ for 1 hour at room temperature. The resulting precipitate was filtered and dried in vacuo to give 9 as colorless crystals (38.6 g, 93\%).

Mp 85 86 ${ }^{\circ} \mathrm{C}$; IR (KBr) cm $\mathrm{cm}^{-1} 1613,1343,1193,1126$, 1041, 949; ${ }^{1} \mathrm{H}-\mathrm{NMR}\left(270 \mathrm{MHz}, \mathrm{CDCl}_{3}\right) \delta 3.07(3 \mathrm{H}, \mathrm{s})$, $3.38(2 \mathrm{H}, \mathrm{t}, J=7.6 \mathrm{~Hz}), 4.03(2 \mathrm{H}, \mathrm{t}, J=7.6 \mathrm{~Hz}), 4.14 \sim 4.19$ $(2 \mathrm{H}, \mathrm{m}), 4.30 \sim 4.37(2 \mathrm{H}, \mathrm{m}), 5.26 \sim 5.32(1 \mathrm{H}, \mathrm{m})$; HRMS (EI) calcd for $\mathrm{C}_{7} \mathrm{H}_{12} \mathrm{~N}_{2} \mathrm{O}_{3} \mathrm{~S}_{2} 236.0289$, found $\mathrm{m} / \mathrm{z} 236.0286$ $(\mathrm{M})^{+}$.

\section{3-Acetylthio-1-(1,3-thiazolin-2-yl)azetidine (10)}

To DMF $(44 \mathrm{ml})$ at $100^{\circ} \mathrm{C}$ were added 3-methanesulfonyloxy-1-(1,3-thiazolin-2-yl)azetidine $\quad(\mathbf{9}, \quad 5.14 \mathrm{~g}$, $21.8 \mathrm{mmol})$ and potassium thioacetate $(3.73 \mathrm{~g}, 32.7 \mathrm{mmol})$, and the mixture was stirred for 5.5 hours at $100^{\circ} \mathrm{C}$. The reaction mixture was concentrated under reduced pressure, and then AcOEt $(30 \mathrm{ml})$ and water $(20 \mathrm{ml})$ were added to the residue. The AcOEt layer was separated, and the aqueous layer was extracted with AcOEt $(8 \mathrm{ml})$ three times. The combined organic layer was washed with brine, dried over $\mathrm{MgSO}_{4}$ and the filtrate was concentrated under reduced pressure to give $\mathbf{1 0}$ as yellow oil $(4.1 \mathrm{~g}, 88 \%)$.

IR $(\mathrm{KBr}) \mathrm{cm}^{-1}$ 2944, 2869, 1694, 1616, 1355, 1296, 1132, 952; ${ }^{1} \mathrm{H}-\mathrm{NMR}\left(270 \mathrm{MHz}, \mathrm{CDCl}_{3}\right) \delta 2.34(3 \mathrm{H}, \mathrm{s})$, $3.36(2 \mathrm{H}, \mathrm{t}, J=7.4 \mathrm{~Hz}), 3.87 \sim 4.92(2 \mathrm{H}, \mathrm{m}), 4.00(2 \mathrm{H}, \mathrm{t}$, $J=7.4 \mathrm{~Hz}), 4.30 \sim 4.35(1 \mathrm{H}, \mathrm{m}), 4.40 \sim 4.46(2 \mathrm{H}, \mathrm{m})$; HRMS (EI) calcd for $\mathrm{C}_{8} \mathrm{H}_{12} \mathrm{~N}_{2} \mathrm{OS}_{2} 216.0391$, found $\mathrm{m} / \mathrm{z}$ $216.0380(\mathrm{M})^{+}$; Anal. Calcd for $\mathrm{C}_{8} \mathrm{H}_{12} \mathrm{~N}_{2} \mathrm{OS}_{2}$ : C, 44.42; H, 5.59; N, 12.95. Found: C, 44.17; H, 5.64; N, 12.84 .

\section{3-Mercapto-1-(1,3-thiazolin-2-yl)azetidine hydrochloride (1)}

To a solution of 3-acetylthio-1-(1,3-thiazolin-2-yl)azetidine $(\mathbf{1 0}, 52.1 \mathrm{~g}, 241 \mathrm{mmol})$ in IPA $(24.1 \mathrm{ml})$ was added $2.0 \mathrm{~mol} /$ liter $\mathrm{KOH}$ in $\mathrm{MeOH}(133 \mathrm{ml}, 265 \mathrm{mmol})$ under $5^{\circ} \mathrm{C}$. After being stirred for 10 minutes at $5^{\circ} \mathrm{C}, 2.0 \mathrm{M} \mathrm{HCl}$ in $\mathrm{MeOH}(289 \mathrm{ml}, 578 \mathrm{mmol})$ was added and the mixture 
was stirred for additional 15 minutes. The insoluble material was filtered off, and the filtrate was concentrated under reduced pressure. The residue was purified by crystallization from MeCN $(48 \mathrm{ml})$ and THF $(289 \mathrm{ml})$ to give $\mathbf{1}$ as colorless crystals ( $44.4 \mathrm{~g}, 87 \%$ ).

Mp 134 136 ${ }^{\circ} \mathrm{C}$; IR (KBr) $\mathrm{cm}^{-1} 2960,2430,1639$, $1135 ;{ }^{1} \mathrm{H}-\mathrm{NMR} \quad\left(270 \mathrm{MHz}, \mathrm{CDCl}_{3}\right) \quad \delta 2.57 \quad(1 \mathrm{H}, \mathrm{d}$, $J=8.2 \mathrm{~Hz}), 3.59(2 \mathrm{H}, \mathrm{t}, J=7.4 \mathrm{~Hz}), 4.02 \sim 4.18(4 \mathrm{H}, \mathrm{m})$, $4.63(2 \mathrm{H}, \mathrm{t}, J=7.4 \mathrm{~Hz}), 5.19 \sim 5.26(1 \mathrm{H}, \mathrm{m}), 12.19(1 \mathrm{H}, \mathrm{s})$; HRMS (FAB) calcd for $\mathrm{C}_{6} \mathrm{H}_{11} \mathrm{~N}_{2} \mathrm{~S}_{2}$ 175.0364, found $\mathrm{m} / \mathrm{z}$ $175.0376(\mathrm{M}-\mathrm{Cl})^{+}$; Anal. Calcd for $\mathrm{C}_{6} \mathrm{H}_{11} \mathrm{ClN}_{2} \mathrm{~S}_{2}$ : C, 34.19; H, 5.26; N, 13.29. Found: C, 34.17; H, 5.18; N, 13.24.

\section{4-Nitrobenzyl $(1 R, 5 S, 6 S)-6-[(R)-1-h y d r o x y e t h y l]-1-$ methyl-2-[1-(1,3-thiazolin-2-yl)azetidin-3-yl]thio-1- carbapen-2-em-3-carboxylate (3)}

To a suspension of 3-mercapto-1-(1,3-thiazolin-2yl)azetidine hydrochloride $(1,5.79 \mathrm{~g}, 27.5 \mathrm{mmol})$ and 4 nitrobenzyl $(1 R, 5 R, 6 S)$-2-diphenylphosphoryloxy-6-[(R)-1hydroxyethyl]-1-methyl-1-carbapen-2-em-3-carboxylate (2, $14.86 \mathrm{~g}, 25 \mathrm{mmol})$ in dry $\mathrm{MeCN}(100 \mathrm{ml})$ was added diisopropylethylamine $(9.58 \mathrm{ml}, 55 \mathrm{mmol})$ under $-20^{\circ} \mathrm{C}$. After being stirred for 2 hours at $-20^{\circ} \mathrm{C}$ under a nitrogen atmosphere, the reaction mixture was quenched with water $(60 \mathrm{ml})$ and stirred at $5^{\circ} \mathrm{C}$ for 0.5 hours. The resulting precipitate was filtered, washed successively with $\mathrm{MeCN}$ water $(1: 1,30 \mathrm{ml})$ and IPA $(30 \mathrm{ml})$ and then dried in vacuo to obtain 3 as colorless crystals (12.3 g, 94\%).

Mp $178 \sim 180^{\circ} \mathrm{C}$; IR $(\mathrm{KBr}) \mathrm{cm}^{-1} 2939,1768,1702$, 1612，1553，1513，1340，1140; ${ }^{1} \mathrm{H}-\mathrm{NMR} \quad(400 \mathrm{MHz}$, $\left.\mathrm{CDCl}_{3}\right) \delta 1.24(3 \mathrm{H}, \mathrm{d}, J=7.3 \mathrm{~Hz}), 1.36(3 \mathrm{H}, \mathrm{d}, J=6.3 \mathrm{~Hz})$, $3.15 \sim 3.19(1 \mathrm{H}, \mathrm{m}), 3.27(1 \mathrm{H}, \mathrm{dd}, J=2.6,6.8 \mathrm{~Hz}), 3.37$ $(2 \mathrm{H}, \mathrm{t}, J=7.5 \mathrm{~Hz}), 3.94 \sim 3.98(2 \mathrm{H}, \mathrm{m}), 4.01(2 \mathrm{H}, \mathrm{t}$, $J=7.5 \mathrm{~Hz}), 4.11 \sim 4.15(1 \mathrm{H}, \mathrm{m}), 4.21 \sim 4.28(2 \mathrm{H}, \mathrm{m})$, $4.35 \sim 4.40(2 \mathrm{H}, \mathrm{m}), 5.25(1 \mathrm{H}, \mathrm{d}, J=13.7 \mathrm{~Hz}), 5.51(1 \mathrm{H}, \mathrm{d}$, $J=13.7 \mathrm{~Hz}), 7.66(2 \mathrm{H}, \mathrm{d}, J=8.7 \mathrm{~Hz}), 8.23(2 \mathrm{H}, \mathrm{d}$, $J=8.7 \mathrm{~Hz}$ ); HRMS (FAB) calcd for $\mathrm{C}_{23} \mathrm{H}_{27} \mathrm{~N}_{4} \mathrm{O}_{6} \mathrm{~S}_{2}$ 519.1372, found $\mathrm{m} / z$ 519.1391 $(\mathrm{M}+\mathrm{H})^{+}$; Anal. Calcd for $\mathrm{C}_{23} \mathrm{H}_{26} \mathrm{~N}_{4} \mathrm{O}_{6} \mathrm{~S}_{2}: \mathrm{C}, 53.27$; H, 5.05; N, 10.80. Found: C, 53.36; H, 5.15; N, 10.76 .

$(1 R, 5 S, 6 S)-6-[(R)-1-H y d r o x y e t h y l]-1-m e t h y l-2-[1-(1,3-$ thiazolin-2-yl)azetidin-3-yl]thio-1-carbapen-2-em-3carboxylic acid (4)

To a mixture of $\mathbf{3}(9.33 \mathrm{~g}, 18 \mathrm{mmol})$ and sodium hydrogen carbonate $(756 \mathrm{mg}, 9 \mathrm{mmol})$ in $n-\mathrm{BuOH}(126 \mathrm{ml})$ were added $10 \%$ Pd-C $(1.4 \mathrm{~g}$, dry reduced) and water $(156 \mathrm{ml})$, and the mixture was stirred vigorously for 1.5 hours under $400 \mathrm{kPa}$ pressure of hydrogen at room temperature. The catalyst was removed by filtration, and the $\mathrm{pH}$ of the filtrate was adjusted to 5.6 using $1.0 \mathrm{M}$ aqueous $\mathrm{HCl}$. After separation, the aqueous layer was poured into cold acetone $(467 \mathrm{ml})$ at $5^{\circ} \mathrm{C}$, and the mixture was stirred for 10 minutes. Additional acetone $(467 \mathrm{ml})$ was added dropwise over 20 minutes and the mixture was stirred for 3 hours at $5^{\circ} \mathrm{C}$. The resulting precipitate was filtered and dried in vacuo to give tetrahydrate of $\mathbf{4}$ as colorless crystals $(6.79 \mathrm{~g}, 82 \%)$.

$\mathrm{Mp} 170^{\circ} \mathrm{C}$ (decomp.); IR (KBr) cm $\mathrm{cm}^{-1} 1736,1649,1573$; ${ }^{1} \mathrm{H}-\mathrm{NMR}\left(400 \mathrm{MHz}, \mathrm{D}_{2} \mathrm{O}\right) \delta 1.16(3 \mathrm{H}, \mathrm{d}, J=7.2 \mathrm{~Hz}), 1.28$ $(3 \mathrm{H}, \mathrm{d}, J=6.4 \mathrm{~Hz}), 3.18(1 \mathrm{H}, \mathrm{dq}, J=7.2,9.0 \mathrm{~Hz}), 3.42(1 \mathrm{H}$, dd, $J=2.5,6.2 \mathrm{~Hz}), 3.63(2 \mathrm{H}, \mathrm{t}, J=7.5 \mathrm{~Hz}), 3.99(2 \mathrm{H}, \mathrm{t}$, $J=7.5 \mathrm{~Hz}), 4.17 \sim 4.25(4 \mathrm{H}, \mathrm{m}), 4.31 \sim 4.38(1 \mathrm{H}, \mathrm{m})$, 4.70 4.79 (2H, m); HRMS (FAB) calcd for $\mathrm{C}_{16} \mathrm{H}_{22} \mathrm{~N}_{3} \mathrm{O}_{4} \mathrm{~S}_{2}$ 384.1052, found $m / z 384.1045(\mathrm{M}+\mathrm{H})^{+}$; Anal. Calcd for $\mathrm{C}_{16} \mathrm{H}_{21} \mathrm{~N}_{3} \mathrm{O}_{4} \mathrm{~S}_{2} \cdot 4 \mathrm{H}_{2} \mathrm{O}: \mathrm{C}, 42.18 ; \mathrm{H}, 6.37$;, 9.21 . Found: $\mathrm{C}$, 42.07; H, 6.11; N, 9.01.

\section{Typical Procedure for Esterification of 4 (Scheme 3):} Pivaloyloxymethyl $(1 R, 5 S, 6 S)-6-[(R)-1-h y d r o x y e t h y l]-1-$ methyl-2-[1-(1,3-thiazolin-2-yl)azetidin-3-yl]thio-1carbapen-2-em-3-carboxylate (5)

To a solution of the tetrahydrate of $4(445 \mathrm{mg}, 1 \mathrm{mmol})$, benzyltriethylammonium chloride $(456 \mathrm{mg}, 2 \mathrm{mmol})$ and chloromethyl pivalate $(301 \mathrm{mg}, 2 \mathrm{mmol})$ in DMF $(1 \mathrm{ml})$ was added diisopropylethylamine $(0.44 \mathrm{ml}, 2 \mathrm{mmol})$ and the mixture was stirred for 4 hours at $45^{\circ} \mathrm{C}$. After cooling to $5^{\circ} \mathrm{C}$, AcOEt $(2 \mathrm{ml})$ and water $(2 \mathrm{ml})$ were added and then the mixture was adjusted to $\mathrm{pH} 4$ by using $1.0 \mathrm{M}$ aqueous citric acid. After discarding organic layer, potassium hydrogen carbonate was added to adjust $\mathrm{pH}$ to 7.6. The mixture was extracted with AcOEt, and the organic layer was washed with water and brine, dried over $\mathrm{MgSO}_{4}$ and concentrated under reduced pressure. The residue was purified by silica gel column chromatography (EtOH $\mathrm{CH}_{2} \mathrm{Cl}_{2}$-Acetone, $1: 20: 40$ ) to yield $\mathbf{5}$ as a yellow amorphous solid. The amorphous solid was triturated with AcOEt to give 5 as colorless crystals (388 mg, 78\%).

Mp $140 \sim 142^{\circ} \mathrm{C}$; IR $(\mathrm{KBr}) \mathrm{cm}^{-1} 1778,1753,1609 ;{ }^{1} \mathrm{H}-$ NMR $\left(270 \mathrm{MHz}, \mathrm{CDCl}_{3}\right) \delta 1.22(3 \mathrm{H}, \mathrm{d}, J=7.2 \mathrm{~Hz}), 1.22$ $(9 \mathrm{H}, \mathrm{s}), 1.31(3 \mathrm{H}, \mathrm{d}, J=6.3 \mathrm{~Hz}), 3.17(1 \mathrm{H}, \mathrm{dq}, J=7.2$, $9.2 \mathrm{~Hz}), 3.22(1 \mathrm{H}, \mathrm{dd}, J=2.7,6.3 \mathrm{~Hz}), 3.37(2 \mathrm{H}, \mathrm{t}$, $J=7.2 \mathrm{~Hz}), 3.94(2 \mathrm{H}, \mathrm{m}), 4.00(2 \mathrm{H}, \mathrm{m}), 4.11 \sim 4.23(3 \mathrm{H}$, m), $4.35 \sim 4.43(2 \mathrm{H}, \mathrm{m}), 5.83(1 \mathrm{H}, \mathrm{d}, J=5.3 \mathrm{~Hz}), 5.96(1 \mathrm{H}$, d, $J=5.3 \mathrm{~Hz}$ ); HRMS (EI) calcd for $\mathrm{C}_{22} \mathrm{H}_{31} \mathrm{~N}_{3} \mathrm{O}_{6} \mathrm{~S}_{2}$ 497.1654, found $\mathrm{m} / \mathrm{z} 497.1660(\mathrm{M})^{+}$; Anal. Calcd for $\mathrm{C}_{22} \mathrm{H}_{31} \mathrm{~N}_{3} \mathrm{O}_{6} \mathrm{~S}_{2}: \mathrm{C}, 53.10 ; \mathrm{H}, 6.28 ; \mathrm{N}, 8.44$. Found: C, 53.08; $\mathrm{H}, 6.19 ; \mathrm{N}, 8.41$. 
4-Methylcyclohexylcarbonyloxymethyl $(1 R, 5 S, 6 S)-6-$ [(R)-1-hydroxyethyl]-1-methyl-2-[1-(1,3-thiazolin-2-yl)azetidin-3-yl]thio-1-carbapen-2-em-3-carboxylate (11) Amorphous powder ( $80 \%$ yield), mp $78 \sim 79^{\circ} \mathrm{C}$; IR ( $\left.\mathrm{KBr}\right)$ $\mathrm{cm}^{-1}$ 3382, 2928, 1774, 1615, 1545; ${ }^{1} \mathrm{H}-\mathrm{NMR}(270 \mathrm{MHz}$, $\left.\mathrm{CDCl}_{3}\right) \delta 0.88(0.9 \mathrm{H}, \mathrm{d}, J=6.6 \mathrm{~Hz}), 0.89(2.1 \mathrm{H}, \mathrm{d}$, $J=6.6 \mathrm{~Hz}), 1.22(3 \mathrm{H}, \mathrm{d}, J=7.3 \mathrm{~Hz}), 1.34(3 \mathrm{H}, \mathrm{d}, J=6.3 \mathrm{~Hz})$, $1.34 \sim 1.80(7 \mathrm{H}, \mathrm{m}), 1.96 \sim 2.05(2 \mathrm{H}, \mathrm{m}), 2.23 \sim 2.28(0.3 \mathrm{H}$, m), $2.45(1 \mathrm{H}$, br s), $2.57 \sim 2.59(0.7 \mathrm{H}, \mathrm{m}), 3.15 \sim 3.21(1 \mathrm{H}$, $\mathrm{m}), 3.23(1 \mathrm{H}, \mathrm{dd}, J=2.3,6.6 \mathrm{~Hz}), 3.40(2 \mathrm{H}, \mathrm{t}, J=7.6 \mathrm{~Hz})$, $4.00 \sim 4.05$ (4H, m), 4.15 4.27 (3H, m), 4.43 4.51 $(2 \mathrm{H}$, $\mathrm{m}), 5.86(0.3 \mathrm{H}, \mathrm{d}, J=5.6 \mathrm{~Hz}), 5.87(0.7 \mathrm{H}, \mathrm{d}, J=5.6 \mathrm{~Hz})$, $5.94(0.3 \mathrm{H}, \mathrm{d}, J=5.6 \mathrm{~Hz}), 5.95(0.7 \mathrm{H}, \mathrm{d}, J=5.6 \mathrm{~Hz})$; HRMS (EI) calcd for $\mathrm{C}_{25} \mathrm{H}_{35} \mathrm{~N}_{3} \mathrm{O}_{6} \mathrm{~S}_{2} 537.1967$, found $\mathrm{m} / z 537.1974$ $(\mathrm{M})^{+}$.

1-(Cyclohexyloxycarbonyloxy)ethyl $(1 R, 5 S, 6 S)-6-[(R)-1-$ hydroxyethyl]-1-methyl-2-[1-(1,3-thiazolin-2-yl)azetidin3-yl]thio-1-carbapen-2-em-3-carboxylate (12)

Amorphous powder ( $82 \%$ yield, diastereo-mixture), mp $107 \sim 109^{\circ} \mathrm{C}$; IR (KBr) cm ${ }^{-1} 3184,2937,1790,1611,1548$; ${ }^{1} \mathrm{H}-\mathrm{NMR}\left(270 \mathrm{MHz}, \mathrm{CDCl}_{3}\right) \delta 1.20 \sim 1.95(19 \mathrm{H}, \mathrm{m})$, $3.12 \sim 3.23(2 \mathrm{H}, \mathrm{m}), 3.36(2 \mathrm{H}, \mathrm{t}, J=7.6 \mathrm{~Hz}), 3.92 \sim 4.03$ $(4 \mathrm{H}, \mathrm{m}), 4.10 \sim 4.23(3 \mathrm{H}, \mathrm{m}), 4.34 \sim 4.42(2 \mathrm{H}, \mathrm{m})$, $4.63 \sim 4.69(1 \mathrm{H}, \mathrm{m}), 6.88(1 \mathrm{H}, \mathrm{q}, J=2.6 \mathrm{~Hz})$; HRMS (FAB) calcd for $\mathrm{C}_{25} \mathrm{H}_{36} \mathrm{~N}_{3} \mathrm{O}_{7} \mathrm{~S}_{2}$ 554.1995, found $m / z$ 554.1964 $(\mathrm{M}+\mathrm{H})^{+}$; Anal. Calcd for $\mathrm{C}_{25} \mathrm{H}_{35} \mathrm{~N}_{3} \mathrm{O}_{7} \mathrm{~S}_{2}$ : C, 54.23; H, 6.37; $\mathrm{N}, 7.59$. Found: C, 53.72; H, 6.27; N, 7.99.

1-Methylcyclohexylcarbonyloxymethyl $(1 R, 5 S, 6 S)-6-$ [(R)-1-hydroxyethyl]-1-methyl-2-[1-(1,3-thiazolin-2-yl)azetidin-3-yl]thio-1-carbapen-2-em-3-carboxylate (13) Colorless crystals $(88 \%$ yield from $\mathrm{MeCN}), \mathrm{mp}$ $137 \sim 139^{\circ} \mathrm{C}$; IR (KBr) cm $\mathrm{cm}^{-1} 3123,2940,1790,1747,1717$, 1613 1550; ${ }^{1} \mathrm{H}-\mathrm{NMR}\left(270 \mathrm{MHz}, \mathrm{CDCl}_{3}\right) \delta 1.13 \sim 1.54(8 \mathrm{H}$, m), $1.19(3 \mathrm{H}, \mathrm{s}), 1.22(3 \mathrm{H}, \mathrm{d}, J=7.3 \mathrm{~Hz}), 1.33(3 \mathrm{H}, \mathrm{d}$, $J=6.3 \mathrm{~Hz}), 2.02 \sim 2.11(2 \mathrm{H}, \mathrm{m}), 2.41(1 \mathrm{H}, \mathrm{br} \mathrm{s}), 3.13 \sim 3.25$ $(2 \mathrm{H}, \mathrm{m}), 3.39(2 \mathrm{H}, \mathrm{t}, J=7.6 \mathrm{~Hz}), 3.97 \sim 4.05(4 \mathrm{H}, \mathrm{m})$, $4.11 \sim 4.26(3 \mathrm{H}, \mathrm{m}), 4.42 \sim 4.49(2 \mathrm{H}, \mathrm{m}), 5.87(1 \mathrm{H}, \mathrm{d}$, $J=5.6 \mathrm{~Hz}), 5.97(1 \mathrm{H}, \mathrm{d}, J=5.6 \mathrm{~Hz})$; HRMS (FAB) calcd for $\mathrm{C}_{25} \mathrm{H}_{36} \mathrm{~N}_{3} \mathrm{O}_{6} \mathrm{~S}_{2}$ 538.2046, found $m / z$ 538.2032 $(\mathrm{M}+\mathrm{H})^{+}$; Anal. Calcd for $\mathrm{C}_{25} \mathrm{H}_{35} \mathrm{~N}_{3} \mathrm{O}_{6} \mathrm{~S}_{2}$ : C, 55.84; H, 6.56; N, 7.81. Found: C, 55.52; H, 6.47; N, 7.65.

Cyclohexylacetoxymethyl $(1 R, 5 S, 6 S)-6-[(R)-1-$ hydroxyethyl]-1-methyl-2-[1-(1,3-thiazolin-2-yl)azetidin3-yl]thio-1-carbapen-2-em-3-carboxylate (14)

Amorphous powder ( $76 \%$ yield), mp $70 \sim 71{ }^{\circ} \mathrm{C}$; IR (KBr) $\mathrm{cm}^{-1} 3381,2925,1771,1615,1542 ;{ }^{1} \mathrm{H}-\mathrm{NMR}(270 \mathrm{MHz}$, $\left.\mathrm{CDCl}_{3}\right) \delta 0.81 \sim 1.00(2 \mathrm{H}, \mathrm{m}), 1.00 \sim 1.26(3 \mathrm{H}, \mathrm{m}), 1.15$ $(3 \mathrm{H}, \mathrm{d}, J=7.3 \mathrm{~Hz}), 1.25(3 \mathrm{H}, \mathrm{d}, J=6.3 \mathrm{~Hz}), 1.55 \sim 1.80(6 \mathrm{H}$, m), $2.19(2 \mathrm{H}, \mathrm{d}, J=6.9 \mathrm{~Hz}), 3.10(1 \mathrm{H}$, quint, $J=7.3 \mathrm{~Hz})$, $3.15(1 \mathrm{H}, \mathrm{dd}, J=2.3,6.9 \mathrm{~Hz}), 3.28(1 \mathrm{H}, \mathrm{br} \mathrm{s}), 3.31(2 \mathrm{H}, \mathrm{t}$, $J=7.6 \mathrm{~Hz}), 3.87 \sim 3.91(2 \mathrm{H}, \mathrm{m}), 3.94(2 \mathrm{H}, \mathrm{t}, J=7.6 \mathrm{~Hz})$, $4.04 \sim 4.16(3 \mathrm{H}, \mathrm{m}), 4.30 \sim 4.37(2 \mathrm{H}, \mathrm{m}), 5.79(1 \mathrm{H}, \mathrm{d}$, $J=5.6 \mathrm{~Hz}), 5.85(1 \mathrm{H}, \mathrm{d}, J=5.6 \mathrm{~Hz})$; HRMS (EI) calcd for $\mathrm{C}_{25} \mathrm{H}_{35} \mathrm{~N}_{3} \mathrm{O}_{6} \mathrm{~S}_{2}$ 537.1967, found $m / z 537.1992(\mathrm{M})^{+}$.

Cyclohexylcarbonyloxymethyl $(1 R, 5 S, 6 S)-6-[(R)-1-$ hydroxyethyl]-1-methyl-2-[1-(1,3-thiazolin-2-yl)azetidin3-yl]thio-1-carbapen-2-em-3-carboxylate (15)

Colorless crystals $(71 \%$ yield from AcOEt $), \mathrm{mp}$ $120 \sim 122^{\circ} \mathrm{C}$; IR $(\mathrm{KBr}) \mathrm{cm}^{-1} 3195,2931,1785,1695,1611$, $1541 ;{ }^{1} \mathrm{H}-\mathrm{NMR}\left(270 \mathrm{MHz}, \mathrm{CDCl}_{3}\right) \quad \delta \quad 1.31 \quad(3 \mathrm{H}, \mathrm{d}$, $J=7.3 \mathrm{~Hz}), 1.41(3 \mathrm{H}, \mathrm{d}, J=6.3 \mathrm{~Hz}), 1.31 \sim 1.73(6 \mathrm{H}, \mathrm{m})$, $1.81 \sim 1.85(2 \mathrm{H}, \mathrm{m}), 1.99 \sim 2.13(2 \mathrm{H}, \mathrm{m}), 2.40 \sim 2.51(1 \mathrm{H}$, m), $2.99(1 \mathrm{H}$, br s), $3.12 \sim 3.18(1 \mathrm{H}, \mathrm{m}), 3.20 \sim 3.27(1 \mathrm{H}$, m), $3.47(2 \mathrm{H}, \mathrm{t}, J=7.6 \mathrm{~Hz}), 4.03 \sim 4.13(4 \mathrm{H}, \mathrm{m}), 4.16 \sim 4.34$ $(3 \mathrm{H}, \mathrm{m}), 4.51(2 \mathrm{H}, \mathrm{t}, J=7.6 \mathrm{~Hz}), 5.93(1 \mathrm{H}, \mathrm{d}, J=5.6 \mathrm{~Hz})$, $6.03(1 \mathrm{H}, \quad \mathrm{d}, J=5.6 \mathrm{~Hz})$; HRMS (EI) calcd for $\mathrm{C}_{24} \mathrm{H}_{33} \mathrm{~N}_{3} \mathrm{O}_{6} \mathrm{~S}_{2}$ 523.1811, found $\mathrm{m} / z$ 523.1791 (M) ${ }^{+}$; Anal. Calcd for $\mathrm{C}_{24} \mathrm{H}_{33} \mathrm{~N}_{3} \mathrm{O}_{6} \mathrm{~S}_{2}:$ C, 55.05; H, 6.35; N, 8.02. Found: C, 55.00; H, 6.33; N, 7.91.

(5-Methyl-2-oxo-1,3-dioxolen-4-yl)methyl $(1 R, 5 S, 6 S)-6-$ [(R)-1-hydroxyethyl]-1-methyl-2-[1-(1,3-thiazolin-2-yl)azetidin-3-yl]thio-1-carbapen-2-em-3-carboxylate (16) Amorphous powder (54\% yield), mp $115^{\circ} \mathrm{C}$ (decomp.); IR $(\mathrm{KBr}) \mathrm{cm}^{-1} 3382,2969,1820,1767,1610,1457 ;{ }^{1} \mathrm{H}-\mathrm{NMR}$ $\left(270 \mathrm{MHz}, \mathrm{CDCl}_{3}\right) \delta 1.22(3 \mathrm{H}, \mathrm{d}, J=7.3 \mathrm{~Hz}), 1.34(3 \mathrm{H}, \mathrm{d}$, $J=6.3 \mathrm{~Hz}), 2.22(3 \mathrm{H}, \mathrm{s}), 2.46(1 \mathrm{H}, \mathrm{br} \mathrm{s}), 3.14 \sim 3.26(2 \mathrm{H}$, m), $3.39 \sim 3.45(2 \mathrm{H}, \mathrm{m}), 4.00 \sim 4.09(4 \mathrm{H}, \mathrm{m}), 4.13 \sim 4.28$ $(3 \mathrm{H}, \mathrm{m}), 4.47 \sim 4.56(2 \mathrm{H}, \mathrm{m}), 4.98(1 \mathrm{H}, \mathrm{d}, J=3.9 \mathrm{~Hz}), 5.05$ $\left(1 \mathrm{H}, \mathrm{d}, J=3.9 \mathrm{~Hz}\right.$ ); HRMS (FAB) calcd for $\mathrm{C}_{21} \mathrm{H}_{26} \mathrm{~N}_{3} \mathrm{O}_{7} \mathrm{~S}_{2}$ 496.1212, found $m / z 496.1240(\mathrm{M}+\mathrm{H})^{+}$.

1,3-Dihydro-3-oxo-1-isobenzofuranyl $(1 R, 5 S, 6 S)-6-[(R)-$ 1-hydroxyethyl]-1-methyl-2-[1-(1,3-thiazolin-2-yl)azetidin-3-yl]thio-1-carbapen-2-em-3-carboxylate (17) Amorphous powder (84\% yield), mp $120^{\circ} \mathrm{C}$ (decomp.); IR $(\mathrm{KBr}) \mathrm{cm}^{-1}$ 3421, 2969, 1782, 1611, 1541; ${ }^{1} \mathrm{H}-\mathrm{NMR}$ $\left(270 \mathrm{MHz}, \mathrm{CDCl}_{3}\right) \delta 1.21(1.5 \mathrm{H}, \mathrm{d}, J=7.3 \mathrm{~Hz}), 1.24(1.5 \mathrm{H}$, d, $J=6.9 \mathrm{~Hz}), 1.29(3 \mathrm{H}, \mathrm{d}, J=6.3 \mathrm{~Hz}), 2.74(1 \mathrm{H}$, br s$)$, $3.18 \sim 3.28(2 \mathrm{H}, \mathrm{m}), 3.34 \sim 3.41(2 \mathrm{H}, \mathrm{m}), 3.94 \sim 4.04(4 \mathrm{H}$, $\mathrm{m}), 4.10 \sim 4.26(3 \mathrm{H}, \mathrm{m}), 4.39 \sim 4.49(2 \mathrm{H}, \mathrm{m}), 7.45(0.5 \mathrm{H}, \mathrm{s})$, $7.51(0.5 \mathrm{H}, \mathrm{s}), 7.61 \sim 7.79(3 \mathrm{H}, \mathrm{m}), 7.89 \sim 7.94(1 \mathrm{H}, \mathrm{m})$; HRMS (FAB) calcd for $\mathrm{C}_{24} \mathrm{H}_{26} \mathrm{~N}_{3} \mathrm{O}_{6} \mathrm{~S}_{2} 516.1263$, found $\mathrm{m} / \mathrm{z}$ $516.1274(\mathrm{M}+\mathrm{H})^{+}$. 
4-Methylbenzoyloxymethyl $(1 R, 5 S, 6 S)-6-[(R)-1-$ hydroxyethyl]-1-methyl-2-[1-(1,3-thiazolin-2-yl)azetidin3-yl]thio-1-carbapen-2-em-3-carboxylate (18)

Colorless crystals ( $84 \%$ yield from $\mathrm{EtOH}), \mathrm{mp} 91 \sim 93^{\circ} \mathrm{C}$; IR (KBr) cm ${ }^{-1} 3302,2964,1785,1736,1609,1550 ;{ }^{1} \mathrm{H}-$ NMR $\left(270 \mathrm{MHz}, \mathrm{CDCl}_{3}\right) \delta 1.21(3 \mathrm{H}, \mathrm{d}, J=7.3 \mathrm{~Hz}), 1.32$ ( $3 \mathrm{H}, \mathrm{d}, J=6.3 \mathrm{~Hz}$ ), 2.41 (s, 3H), 2.50 (br s, 1H), 3.12 3.24 $(2 \mathrm{H}, \mathrm{m}), 3.38(2 \mathrm{H}, \mathrm{t}, J=7.6 \mathrm{~Hz}), 3.98 \sim 4.04(4 \mathrm{H}, \mathrm{m})$, $4.11 \sim 4.26(3 \mathrm{H}, \mathrm{m}), 4.41 \sim 4.48(2 \mathrm{H}, \mathrm{m}), 6.11(2 \mathrm{H}, \mathrm{d}$, $J=5.6 \mathrm{~Hz}), 6.14(1 \mathrm{H}, \mathrm{d}, J=5.6 \mathrm{~Hz}), 7.24(1 \mathrm{H}, \mathrm{d}, J=8.3 \mathrm{~Hz})$, $7.97(2 \mathrm{H}, \quad \mathrm{d}, J=8.3 \mathrm{~Hz})$; HRMS (FAB) calcd for $\mathrm{C}_{25} \mathrm{H}_{30} \mathrm{~N}_{3} \mathrm{O}_{6} \mathrm{~S}_{2} 532.1576$, found $m / z 532.1584(\mathrm{M}+\mathrm{H})^{+}$.

\section{4-Chlorobenzoyloxymethyl $(1 R, 5 S, 6 S)-6-[(R)-1-$} hydroxyethyl]-1-methyl-2-[1-(1,3-thiazolin-2-yl)azetidin3-yl]thio-1-carbapen-2-em-3-carboxylate (19)

Amorphous powder ( $81 \%$ yield), mp $80 \sim 82^{\circ} \mathrm{C}$; IR $(\mathrm{KBr})$ $\mathrm{cm}^{-1} 3370,2969,2869,1740,1612,1542 ;{ }^{1} \mathrm{H}-\mathrm{NMR}$ $\left(270 \mathrm{MHz}, \mathrm{CDCl}_{3}\right) \delta 1.22(3 \mathrm{H}, \mathrm{d}, J=7.6 \mathrm{~Hz}), 1.32(3 \mathrm{H}, \mathrm{d}$, $J=6.3 \mathrm{~Hz}), 2.63(1 \mathrm{H}, \mathrm{br} \mathrm{s}), 3.15 \sim 3.22(1 \mathrm{H}, \mathrm{m}), 3.23(1 \mathrm{H}$, dd, $J=2.3,6.6 \mathrm{~Hz}), 3.37(2 \mathrm{H}, \mathrm{t}, J=7.4 \mathrm{~Hz}), 3.97 \sim 4.04(4 \mathrm{H}$, $\mathrm{m}), 4.11 \sim 4.26(3 \mathrm{H}, \mathrm{m}), 4.40 \sim 4.46(2 \mathrm{H}, \mathrm{m}), 6.10(1 \mathrm{H}, \mathrm{d}$, $J=5.6 \mathrm{~Hz}), 6.16(1 \mathrm{H}, \mathrm{d}, J=5.6 \mathrm{~Hz}), 7.42(2 \mathrm{H}, \mathrm{d}, J=8.5 \mathrm{~Hz})$, $8.02(2 \mathrm{H}, \mathrm{d}, J=8.5 \mathrm{~Hz})$; HRMS (FAB) calcd for $\mathrm{C}_{24} \mathrm{H}_{27} \mathrm{ClN}_{3} \mathrm{O}_{6} \mathrm{~S}_{2}$ 552.1030, found $m / z 552.0999(\mathrm{M}+\mathrm{H})^{+}$.

\section{Typical Assay Procedure for the Plasma Concentration of 4}

Plasma (100 $\mu$ l) was diluted with an equal volume of $1 \mathrm{M}$ MOPS buffer ( $\mathrm{pH} 7.0)$, and $\mathrm{MeCN}(400 \mu \mathrm{l})$ and $\mathrm{CHCl}_{3}$ ( $800 \mu \mathrm{l})$ were added to the diluted plasma. The mixture was vortex-mixed and then centrifuged. A $20 \mu \mathrm{l}$ sample of upper layer was injected onto the HPLC. HPLC conditions were as follows. Column: Develosil ODS-UG-3 (4.6 by $75 \mathrm{~mm}$ I.D., Nomura Chemical), mobile phase: $0.1 \mathrm{M}$ sodium acetate buffer (pH 5.5) and $\mathrm{MeCN}$ (100:4), flow rate: $1.3 \mathrm{ml} /$ minute, temperature: $35^{\circ} \mathrm{C}$, wavelength: $\lambda=300 \mathrm{~nm}$.

\section{References}

1. Leanza WJ, Wildonger KJ, Miller TW, Christensen BG. $N$ Acetimidoyl- and $\mathrm{N}$-formimidoylthienamycin derivatives: Antipseudomonal $\beta$-lactam antibiotics. J Med Chem 22: 1435-1436 (1979)

2. Miyadera T, Sugimura Y, Hashimoto T, Tanaka T, Iino K, Shibata T, Sugawara S. Synthesis and in vitro activity of a new carbapenem, RS-533. J Antibiot 36: 1034-1039 (1983)

3. Sunagawa M, Matsumura H, Inoue T, Fukasawa M, Kato M. A novel carbapenem antibiotic, SM-7338 structure-activity relationships. J Antibiot 43: 519-532 (1990)
4. Hikida M, Yamazaki Y, Yoshida M, Kawashima K, Nishiki K, Mitsuhashi S. Postantibiotic effect of carbapenems against Pseudomonas aeruginosa. J Antibiot 48: 891-892 (1995)

5. Kumagai T, Tamai S, Abe T, Matsunaga H, Hayashi K, Kishi I, Shiro M, Nagao Y. New straightforward synthesis and characterization of a unique $1 \beta$-methylcarbapenem antibiotic biapenem bearing a $\sigma$-symmetric bicyclotriazoliumthio group as the pendant moiety. J Org Chem 63: 8145-8149 (1998)

6. Sundelof JG, Hajdu R, Gill CJ, Thompson R, Rosen H, Kropp H. Pharmacokinetics of L-749,345, a long-acting carbapenem antibiotic, in primates. Antimicrob Agents Chemother 41: 1743-1748 (1997)

7. Iso $\mathrm{Y}$, Irie $\mathrm{T}$, Iwaki $\mathrm{T}$, Kii $\mathrm{M}$, Sendo $\mathrm{Y}$, Motokawa $\mathrm{K}$, Nishitani Y. Synthesis and modification of a novel $1 \beta$ methylcarbapenem antibiotic, S-4661. J Antibiot 49: 478-484 (1996)

8. Gaviraghi G. Tribactams: a new prospect in $\beta$-lactam chemotherapy. Eur J Med Chem 30 (Suppl.): 467s-478s (1995)

9. Miyauchi M, Endo R, Hisaoka M, Yasuda H, Kawamoto I. Synthesis and structure-activity relationships of a novel oral carbapenem, CS-834. J Antibiot 50: 429-439 (1997)

10. Tanaka M, Kato K, Hakusui H, Murakami Y, Sato K, Ito Y, Kawamoto K. Pharmacokinetics and safety of ascending single doses of DZ-2640, a new oral carbapenem antibiotic, administered to healthy Japanese subjects. Antimicrob Agents Chemother 44: 578-582 (2000)

11. Ferres H. Prodrugs of $\beta$-lactam antibiotics. Drugs of Today 19: 499-538 (1983)

12. Nagao $Y$, Nagase $Y$, Kumagai $T$, Matsunaga $H$, Abe T, Shimada O, Hayashi T, Inoue Y. Asymmetric total synthesis of new non-natural $1 \beta$-methylcarbapenems exhibiting strong antimicrobial activities and stability against human renal dehydropeptidase-I. J Org Chem 57: 4243-4249 (1992)

13. Chatterjee SS, Triggle DJ. Synthesis of azetidin-3-ol. Chem Commun 93 (1968)

14. Burgess K, Ho K. Asymmetric syntheses of all four stereoisomers of 2,3-methanomethionine. J Org Chem 57: 5931-5936 (1992)

15. Lee HW, Kang TW, Kim EN, Shin J, Cha KH, Cho DO, Choi NH, Kim JW, Hong CI. An effective and convenient esterification of cephalosporin derivatives by using quaternary ammonium salts as catalysts. Synthetic Commun 28: 4345-4354 (1998)

16. Hikida M, Itahashi K, Igarashi A, Shiba T, Kitamura M. In vitro antibacterial activity of LJC 11,036, an active metabolite of L-084, a new oral carbapenem antibiotic with potent antipneumococcal activity. Antimicrob Agents Chemother 43: 2010-2016 (1999)

17. Hikida M, Itahashi K, Shiba T, Kitamura M. L-084, a new oral carbapenem: In vitro antibacterial activity. "The 38th Interscience Conference on Antimicrobial Agents and Chemotherapy, San Diego", American Society of Microbiology: Abstract No. F-65 (1998)

18. Kumagai T, Tamai S, Abe T, Hikida M. Current status of oral carbapenem development. Curr Med Chem: AntiInfective Agents 1: 1-14 (2002) 